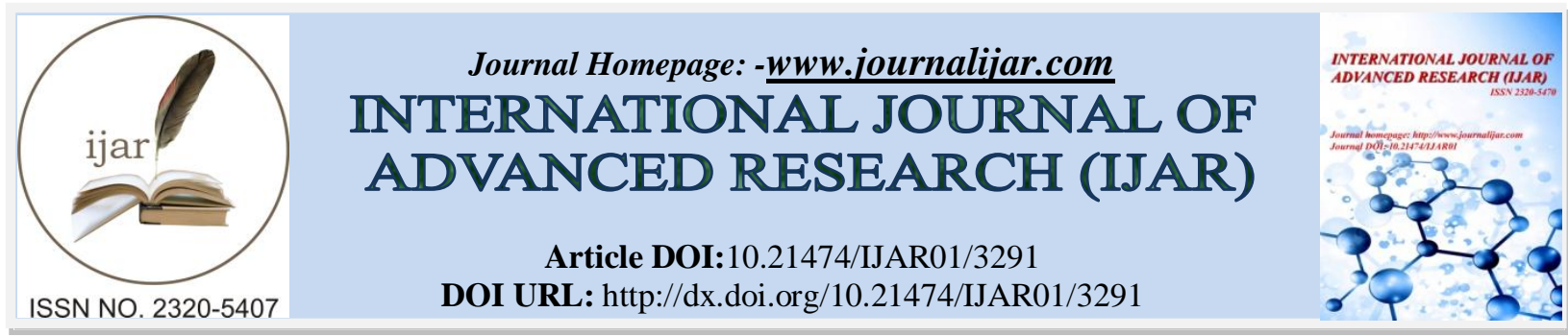

RESEARCH ARTICLE

\title{
TYPE 1 DIABETES MELLITUS AMONG PEDIATRICS AND ADOLESCENTS IN SAUDI ARABIA: A
} SYSTEMATIC REVIEW.

\author{
Mohammed M. Ghandoora ${ }^{1}$, Haneen A. Almutairi ${ }^{1}$, Hanan A. Alsharef ${ }^{1}$, Hassan M. Habis $^{2}$, Ehab O. \\ Mugharbal $^{3}$ and Abdullah M. Albogami ${ }^{4}$. \\ 1. King Fahad General Hospital Jeddah. \\ 2. East Jeddah Hospital. \\ 3. National Guard Hospital Jeddah. \\ 4. King Faisal Specialist Hospital and Research Center Riyadh.
}

\section{Manuscript Info}

Manuscript History

Received: 05 December 2016

Final Accepted: 06 January 2017

Published: February 2017

Key words:-

Type 1DM, Pediatric, Adolescents,

Saudi Arabia, Systematic Review

\section{Abstract}

Introduction: Saudi Arabia is ranked the 7th globally in number of children with T1DM and the 5th regarding the incidence. T1DM sequalae in children and adolescents involve both acute complications such as hypoglycemia attacks, and diabetes ketoacidosis (DKA). Longterm complications microangiopathies in the retina and kidney as well as dyslipidemia were reported.

Objective: we aimed to shed more lights on T1DM among Pediatrics and Adolescent patients in Saudi Arabia in a systematic way.

Methodology: A systematic search of published literature that has addressed T1DM in pediatrics and adolescents was carried out via the internet, using the medical database MEDLINE/PubMed, supported by the US National Library of Medicine. All published articles containing the words "Type 1 Diabetes", "IDDM", "adolescents", "children", "pediatric", in combination with "Saudi Arabia" as part of their title, as a keyword or as a reference in their abstract have been collected. Only full publications and original articles were included in the review process

Results: out of 54 articles, 27 were selected for this review. The number of children with T1DM in KSA is 16,100 cases. The incidence rate is growing by $3 \%$ yearly and Saudi Arabia ranked as 5th in incidence rate. The growth in incidence is driven by environmental factors rather than genetics. The clinical presentation at diagnosis includes polydipsia, polyuria and weight loss with high incidence of DKA. T1DM is commonly associated with autoimmune thyroiditis and coeliac disease. Glycemic control is an area for improvement to reduce both the acute and long term complications. T1DM is associated also with disturbance of lung function, hepatopathy and vitamin D deficiency among T1DM population. Overall, the quality of life for patients and their families were impacted negatively.

Conclusion: in such a devastating disease, more epidemiological studies are needed to screen the environmental factors that trigger the autoimmune response. Awareness campaigns and continuous medical 
education is of utmost importance to detect the disease earlier before development of DKA. Multidisciplinary approach by highly trained diabetes management team should be enforced to improve patients' outcomes.

Copy Right, IJAR, 2017, All rights reserved.

\section{Introduction:-}

Type 1 diabetes mellitus (T1DM) is caused by insulin shortage because of autoimmune disease destroying the $\beta$ beta cells of the pancreas. Globally, there is a worrying growth of T1DM in pediatrics. The trend toward more children developing T1DM has continued and as of 2015, more than half a million children are estimated to be living with type 1 diabetes. Whilst T1DM is much less common compared to type 2, it is still increasing by around 3\% every year, particularly among children. The reasons for this are still unclear, but may be due to variations in ecological risk factors or pattern of viral infections. Around 86,000 children develop T1DM yearly and when insulin is not available, the life expectancy for diseased children is very short. [1]

Saudi Arabia, a country of almost 30 million population of which $26 \%$ are under 14 years old, ranked the 7 th globally in number of children with T1DM.[1] In 2008, Al-Herbish AS et al. reported 109.5/100000 prevalence rate in pediatrics and teenagers.[2] Other forms of autoimmune disorders, such as celiac disease (CD) and thyroiditis (AIT), may increasingly affects T1DM patients.[3,4] Moreover, T1DM sequalae in children and adolescents involve acute complications such as hypoglycemia attacks, and diabetes ketoacidosis (DKA). Long-term complications microangiopathies in the retina and kidney as well as dyslipidemia were reported. [5] In this review, we aimed to shed more lights on T1DM among Pediatrics and Adolescent patients in Saudi Arabia.

\section{Material and Method:-}

A systematic search of published literature that has addressed T1DM in pediatrics and adolescents was carried out via the internet, using the medical database MEDLINE/PubMed, supported by the US National Library of Medicine. The review was conducted to the time of January 2017. The methodology followed by the researcher for the process of selection of articles is presented in. The researchers conducted a review process and the discordances were resolved by consensus. At a first stage, all published articles containing the words "Type 1 Diabetes", "IDDM", "adolescents", "children", "pediatric", in combination with "Saudi Arabia" as part of their title, as a keyword or as a reference in their abstract have been collected. The initial search retrieved 54 articles. A group of filtering conditions was adopted to confine the search results, excluding case reports studies or not original papers. Finally, at the end selection process, the remaining 27 articles were examined in details.

\section{Results:-}

Epidemiology of Type 1 Diabetes Mellitus in SaudiArabia:-

As per diabetes atlas 7th edition, 16100 children suffer from T1DM in Saudi Arabia. Such number ranks Saudi Arabia as 7th globally in terms of numbers and 5th in terms of incidence rate. [1] In 2008, results of nationwide epidemiological survey was published showing that out of 45,682 children and adolescents surveyed, 50 has been identified as having T1DM which brings the prevalence rate to $109.5 / 100,000$. The distribution of prevalence as per region and age group was variable with almost equal gender distribution. [2] The incidence rate showed dramatic increase of numbers over 18 years' study (1990-2007) of children < 15 years old in Saudi Aramco Medical Services Organization. In the 1st 9 years of the study the incidence was 18.05/100,000 and got doubled in the 2nd 9 years to reach 36.99/100,000. [6]

\section{Etiopathogenesis:-}

The exact etiology of type 1 diabetes is unknown. However, it is suggested that it develops in individuals who are genetically susceptible, who come in contact with certain environmental factors, which trigger immune mediated destruction of the $\beta$-cell mass. The interplay between genetics, environmental triggers and immunological response involve a balance between susceptibility and protective factors in each category. [7,8] Among Saudi population, Human Leucocytic Antigen class II DQB $1 * 0201 / 0202-\mathrm{DRB} 1 * 04$ genotype was recognized as inclining to T1DM (P $=0.0002$; odds ratio $=0.67 ; 95 \% \mathrm{CI}=0.009-0.381$ ). On the other hand, high frequency of the DPB $1 * 0401$ may designate a protecting effect of this combination of HLA alleles against T1DM in Saudis. [9]

Despite genetics as a contributing factor to the incidence of Type 1DM, yet it cannot explain the alarming increase in the incidence. Perhaps the environmental factors or triggers like viral infections are changing and leading to this 
leap in incidence. [6] In a community based cross sectional study involving 11552 mothers answering a survey about consanguinity, $6470(56 \%)$ ladies had consanguineous marriage with no significant relation to T1DM (P=.92). [10]

Autoantibodies are responsible for the pathogenesis of T1DM namely islet cell antibodies (ICA), insulin autoantibodies (IAA) and glutamic acid decarboxylase antibodies (GAD). In a study involved 98 children with T1DM in Riyadh between 2000-2007 to identify the prevalence of autoantibodies per age group and correlate between their occurrence and the severity of presenting symptoms. Results showed that among the study population (age range 1-12 years, mean: 6.6 years, equal sex distribution) 49\% presented with diabetic ketoacidosis (DKA). ICA, IAA and GAD were identified in $67 \%, 36 \%$ and $84.4 \%$ respectively. ICA was predominant in preschool children. Children who showed positivity for GAD and ICA but not IAA had more severe clinical presentation. [11]

\section{Clinical Presentation of Type 1 Diabetes Mellitus in Saudi Arabia:-}

Few regional studies reported the clinical presentation of T1DM in Saudi Arabia including patients from Riyadh region in 1991. [12] Eastern provinces in 2001. [13] Al-Medina region in 2004. [14] North West region in 2011,[15] and Riyadh region 2011. [16] The table below summarizes the key presenting symptoms and signs:

Table 1:-Clinical presentation of Type 1 Diabetes Mellitus in Saudi Children and adolescents

\begin{tabular}{|c|c|c|c|c|c|c|c|c|c|c|c|c|}
\hline \multirow{2}{*}{ Region } & \multirow{2}{*}{$\begin{array}{c}\text { Mean Age } \\
\text { in years }\end{array}$} & \multirow{2}{*}{ Male : Female } & \multicolumn{2}{|c|}{ Polydipsia } & \multicolumn{2}{|c|}{ Polyuria } & \multicolumn{2}{|c|}{ Weight loss } & \multicolumn{2}{|c|}{ Abdominal Pain } & DKA & Vomiting \\
\hline & & & & $(\%)$ & $\mathrm{N}$ & $(\%)$ & & $(\%)$ & $\mathrm{N}$ & $(\%)$ & $\mathrm{N} \quad(\%)$ & (\%) \\
\hline Riyadh 110 pts & 5.9 & $51: 59$ & & & & & & & & & $7467.0 \%$ & \\
\hline Eastern 46 pts. & 9 & $1: 1.5$ & 36 & $78.3 \%$ & 31 & $67.4 \%$ & 28 & $60.9 \%$ & 10 & $21.7 \%$ & $3577 \%$ & $10 \quad 21.7 \%$ \\
\hline Al-Madina 230 pts & 6.9 & 111:119 & 221 & $96.0 \%$ & 222 & $96.0 \%$ & 176 & $76.6 \%$ & 118 & $63.4 \%$ & $12755.2 \%$ & $42 \quad 35.3 \%$ \\
\hline NorthWest 311 pts & 6.7 & 152:159 & 304 & $98.0 \%$ & 305 & $98.0 \%$ & 108 & $34.7 \%$ & & & $17255.3 \%$ & \\
\hline Riyadh 369 pts & 12.4 & 175:194 & 310 & $88.8 \%$ & 321 & $92.0 \%$ & 292 & $83.9 \%$ & 172 & $49.3 \%$ & $174 \quad 49.9 \%$ & $14.9 \%$ \\
\hline
\end{tabular}

pts = patients, DKA = Diabetic ketoacidosis

Over all, the cardinal signs and symptoms are polyuria, polydipsia and weight loss whereas diabetic ketoacidosis seems to be less frequent than it is used to be. The time elapsed from the onset of symptoms till presenting to the clinic was few weeks if not days.

\section{Autoimmune diseases Associated with T1DM in Saudi Arabia:-}

Autoimmune thyroiditis whether overt or subclinical was recognized in 14\% of patients.5 Celiac disease was described in 5 publications with varying incidence among T1DM children ranging from $4.9 \%-21.2 \%$ depending on the screening test used and confirmatory biopsy done. [5,17-20]

\section{Management of Type 1 Diabetes Mellitus in Saudi Arabia:-}

Two studies from King Abdul Aziz University (KAAU) hospital described the glycemic control of T1DM in pediatrics and adolescents. [5,21] The protocol for management of T1DM in children at KAAU hospital was outline by the author below. [21]

\section{Insulin Regimens and Self-Monitoring Blood Glucose (SMBG):-}

T1DM children were stratified by age to either conventional insulin regimen for patients $<6$ years or intensive insulin regimen for children $>6$ years and all adolescents. Conventional insulin therapy refers to utilizing 2 injections of premixed insulin /day before meals and guided with SMBG, insulin dosage was adapted per the glycemic control attained. Intensive insulin therapy refers to receiving 3 injections of rapid or short acting insulin /day prior to meals combined with either basal insulin bolus, or insulin pump to compensate for basal insulin.

\section{Diabetes Education and Physical Exercise:-}

Appropriate diabetes education means participation in $\geq 1$ educational session /month. Proper physical exercise should last for $\geq 30$ minutes/day whether indoors and outdoors 3-4 times/week.

The first study, published in 2011, was retrospective single visit that evaluated the glycemic control among T1DM children and adolescents at KAAU hospital from 2006 to 2010. A total of 484 subjects $($ male=213, female= 271), 
mean age was $12.5 \pm 4.1$ years. $38.6 \%$ of the patients were pre-pubertal and $61.4 \%$ pubertal. The overall mean A1c was $9.4 \pm 2.4 \%$ and $31.4 \%$ achieved satisfactory A1c as per American Diabetes Association (ADA) guidelines. The glycemic control of T1DM children in this cohort was less satisfactory because of physical inactivity and patients' educational. The following table depict the main factors contributed to better glycemic control. [21]

Table 2:-Comparison of well and poorly controlled T1DM children and adolescents according to the ADA guidelines [21]

\begin{tabular}{|l|c|c|c|c|}
\hline & Well controlled & Poorly controlled & \multirow{2}{*}{ p value } \\
\cline { 2 - 3 } & $\mathbf{( n = 1 5 2 )}$ & & $\mathbf{( n = 3 3 2})$ & \\
\hline Age (years) & $10.3 \pm 4.1$ & $13.4 \pm 3.6$ & $<0.001 \dagger$ \\
\hline Gender (female) & $89(58.6)$ & & $182(54.8)$ & NS \\
\hline A1c (\%) & $7.2 \pm 0.5$ & & $10.4 \pm 2.3$ & $<0.001$ \\
\hline Duration of T1DM (years) & $2.4 \pm 1.2$ & & $3 \pm 1.3$ & $<0.05$ \\
\hline Insulin regimen (intensive insulin therapy) & $120(79)$ & $314(94.6)$ & $<0.05$ \\
\hline Compliance to educational activities & $128(84.2)$ & $10(3)$ & $<0.001$ \\
\hline SMBG $\geq 4$ times/day & $102(67.1)$ & $85(25.6)$ & $<0.05$ \\
\hline No physical exercise & $61(40.1)$ & $264(79.5)$ & $<0.001$ \\
\hline Exercise (1 to 2/week) & $67(44.1)$ & $57(17.2)$ & $<0.001$ \\
\hline Exercise (3 to 4/week) & $24(15.8)$ & $11(3.3)$ & $<0.001$ \\
\hline $\begin{array}{l}\text { Values are expressed as patients count and percentages (\%) or as mean } \pm \text { SD; } \\
\text { NS: non-significant }\end{array}$ & \\
\hline
\end{tabular}

Later in 2015, a published cross-sectional study had included 228 T1DM subjects attending at the KAAU Hospital, Jeddah, Saudi Arabia from Jan. 2013 to Jan. 2014. Subjects mean age was 10.99 years, and the A1c level was 8.8\%. pre-pubertal age groups had significantly better glycemic control $(\mathrm{p}=0.01)$ compare to pubertal age group. Within this study cohort, acute sequelae included DKA in $65.4 \%$ of patients, and hypoglycemia in $68.9 \%$. Long-term complications were detected in patients including macroangiopathic changes in the retina (4.4\%), microalbuminuria $(16.2 \%)$, and dyslipidemia (8.3\%). [5]

\section{Complications of T1DM in Children and adolescents:-}

Microalbuminuria is a surrogate marker for diabetic nephropathy in T1 DM pediatric patients. Among 409 T1DM children and adolescents the prevalence of microalbuminuria was $11.3 \%$. in patients with T1DM duration $\geq 2$ years, post-pubertal, elevated blood pressure and overweight Microalbuminuria was significantly higher. [22]

\section{Other associated conditions:-}

\section{Pulmonary function:-}

Lung function was evaluated in a group of 52 (26 male and 26 female) volunteer T1DM children within age range from 8-14 years (mean $12.05 \pm 1.42$ years), mean duration of disease of $5.25 \pm 0.47$ years, and mean glycosylated hemoglobin of $11.27 \pm 0.31 \%$. Pulmonary function in children with diabetes showed significant lower mean values of actual lung function parameters forced vital capacity (FVC), peak expiratory flow (PEF), and maximum mid expiratory flow rate (MMEF) compared to their projected standards. [23]

\section{Hepatopathy:-}

In adults, liver disease among diabetics ranges from $17 \%$ to $100 \%$. A pediatric study performed over one year starting Nov. 2008 to evaluate liver diseases among T1DM pediatric subjects. 22 patients (21\%) out of 106 had positive signs of liver affection either hepatomegaly in 10 patients or hyperechogenic liver in 12 patients. Patients with hyperechogenic liver had poorer glycemic control. Upon achieving better glycemic control, hepatopathy was reversible in $60 \%$ of patients at 6 months' follow-up. [24]

\section{Vitamin D Deficiency:-}

In a prospective cross-sectional study evaluating vitamin D deficiency had included 100 Saudi children with T1DM and 100 healthy controls from the pediatrics' department of, Security Forces Hospital, Riyadh, KSA from Jun. to Sept. 2010. 25OHD mean levels were meaningfully lower in the T1DM children compared to the controls $(36.7 \pm$ $14.3 \mathrm{nmol} / \mathrm{l}$ versus $44.8 \pm 14.1 \mathrm{nmol} / \mathrm{l})$. Overall, $84 \%$ of the T1DM children, and $59 \%$ of the control children were vitamin D deficient. [25] 
Another study investigated whether deficiency of vitamin D can impact glycemic control or cardiometabolic complications in T1DM patients KSA. 301 young patients with T1DM in Jeddah during 2010-2013 were enrolled. Only $26.2 \%$ of subjects had a satisfactory A1c level. 25-hydroxyvitamin D [25(OH)D] mean level was 35.15. In males, it was inversely associated with frequency of hypoglycemia $(\mathrm{p}<0.01), \mathrm{BMI}(\mathrm{p}<0.05)$, diastolic blood pressure $(\mathrm{p}<0.05)$, and triglyceride levels $(\mathrm{p}<0.01)$, while in females, it was inversely associated with current age $(\mathrm{p}<0.05)$, age at diagnosis $(\mathrm{p}<0.01)$, and triglyceride levels $(\mathrm{p}<0.01)$. [26]

Health-Related Quality of Life (HRQoL) in T1DM adolescents and their families:In a study involving 315 adolescent patients (12-18 years old) and their caregivers, HRQoL of T1DM and its effect on the caregivers were evaluated. Patients reported a collective mean HRQoL score of 64.8, while caregivers reported significantly lower scores of $60.3(\mathrm{p}=0.003)$. "Worry" subscale had the lowest scores reported by both adolescents and parents. [27]

In another group, 214 adolescents (13-18 years) with T1DM were surveyed to assess HRQoL at Diabetes Treatment Center, Prince Sultan Military Medical City, Riyadh, KSA from Jun. to Sep. 2013. Factors associated with at least one poor HRQoL parameter were Female gender, repeated daily injection, T1DM $>7$ years, DKA and adolescents with poor glycemic control. [28]

\section{Discussion:-}

This is the first review on T1DM children and adolescents in Saudi Arabia. Based on the data we have reviewed regarding the prevalence and incidence of T1DM in KSA we can notice a tremendous increase in the incidence year after year that cannot be explained by genetic factors or high prevalence of consanguinity only and most probably environmental factors and triggers have a principal role in the pathogenesis of T1DM. In comparison to other Arab countries the variability in incidence can be explained by, in addition to infections, other environmental factors particularly change in nutritional habits, including high meat consumption, vitamin D deficiency, low breastfeeding and high intake of nitrites in processed meat might be playing a role as the highest incidence is mainly seen among rich countries. [29]

The cardinal signs and symptoms for T1DM were polydipsia, polyuria and weight loss. There is a trend towards decreased presentation with DKA however in comparison to data from United Kingdom showing DKA among newly diagnosed T1DM is almost 26\%. [30] More effort is needed to diagnose T1DM earlier before development of DKA perhaps in the form of awareness campaigns and continuous medical education.

Several factors are affecting metabolic control among pediatric and adolescent age groups including age of patients, duration of the disease, compliance to SMBG physical exercise and educational activities. Hence a multidisciplinary approach is mandated to improve outcome in our patients. The interaction between poor diabetic control, prevalence of acute and long term diabetes complications, associated autoimmune diseases, associated conditions as well as insulin treatment impact the quality of life not only the patients but also their families and caregivers.

\section{Conclusion:-}

In conclusion, T1DM in pediatrics and adolescents is a devastating disease and more epidemiological studies are needed to screen the environmental factors that trigger the autoimmune response. Awareness campaigns and continuous medical education is of utmost importance to detect the disease earlier before development of DKA. Multidisciplinary approach by highly trained diabetes management team should be enforced to improve the outcome and prevent long term complications.

Author Contributions:-

All authors have equally contributed to the development and review of the manuscript. 


\section{References:-}

1. IDF Diabetes Atlas · Seventh Edition. http://www.diabetesatlas.org/ accessed on January 1st 2017.

2. Al-Herbish AS, El-Mouzan MI, Al-Salloum AA, Al-Qurachi MM, Al-Omar AA. Prevalence of type 1 diabetes mellitus in Saudi Arabian children and adolescents. Saudi Med J 2008; 29: 1285-8.

3. Barker JM, Yu J, Yu L, Wang J, Miao D, Bao F, et al. Autoantibody "subspecificity" in type 1 diabetes. Diabetes Care 2005; 28: 850-55.

4. Hummel S, Hummel M, Banholzer J, Hanak D, Mollenhauer U, Bonifacio E, et al. Development of autoimmunity to transglutaminase $\mathrm{C}$ in children of patients with type 1 diabetes: relationship to islet autoantibodies and infant feeding. Diabetologia 2007; 50: 390-4.

5. Al-Agha AE et al. Glycemic control, complications, and associated autoimmune diseases in children and adolescents with type 1 diabetes in Jeddah, Saudi Arabia. Saudi Med J 2015; Vol. 36 (1): 26-31.

6. Abduljabbar MA et al. Incidence trends of childhood type 1 diabetes in eastern Saudi Arabia. Saudi Med J 2010; Vol. 31 (4): 413-8.

7. Bosi E, Sarugen E. Advances and controversies in aetiopathogenesis of type 1 (insulin dependent) diabetes mellitus. J PediatrEndocrinolMetab 1998; 11 Suppl 293-305.

8. Beressi JP, Djaulah S, Khalil I, Behamamouch S, Bessoud K, Tout M, Hors J, Deschamps O. HLADQA1 and DQB1 study in Algerian type 1 diabetes families. DiabetMetab 1992; 18: 451-8.

9. Al-Hussein KA, Rama NR, Ahmad M, Rozemuller E, Tilanus MG. HLA-DPB1*0401 is associated with dominant protection against type 1 diabetes in the general Saudi population and in subjects with a high-risk DR/DQ haplotype. Eur J Immunogenet. 2003 Apr;30(2):115-9.

10. El Mouzan MI et al. Consanguinity and major genetic disorders in Saudi children: a community-based cross sectional study. Ann Saudi Med 2008; 28(3): 169-73.

11. Al Alwan I, Bin Dajim N, Jawdat D, Tamimi W, Al Ahmdi R, Albuhairan F. Prevalence of autoantibodies in children newly diagnosed with type 1 diabetes mellitus. Br J Biomed Sci. 2012;69(1):31-3.

12. Salman H, Abanamy A, Ghassan B, Khalil M. Childhood diabetes in Saudi Arabia. Diabet Med. 1991 FebMar;8(2):176-8.

13. Kulaylat NA, Narchi H. Clinical picture of childhood type 1 diabetes mellitus in the Eastern Province of Saudi Arabia. Pediatr Diabetes. 2001 Mar;2(1):43-7.

14. Al-Magamsi MS, Habib HS. Clinical presentation of childhood type 1 diabetes mellitus in the Al-Madina region of Saudi Arabia. Pediatr Diabetes. 2004 Jun;5(2):95-8.

15. Habeb AM, Al-Magamsi MS, Halabi S, Eid IM, Shalaby S, Bakoush O. High incidence of childhood type 1 diabetes in Al-Madinah, North West Saudi Arabia (2004-2009). Pediatr Diabetes. 2011 Dec;12(8):676-81.

16. Al Rashed AM. Pattern of presentation in type 1 diabetic patients at the diabetes center of a university hospital. Ann Saudi Med. 2011 May-Jun;31(3):243-9.

17. Al-Ashwal AA, Shabib SM, Sakati NA, Attia NA. Prevalence and characteristics of celiac disease in type I diabetes mellitus in Saudi Arabia. Saudi Med J. 2003 Oct;24(10):1113-5.

18. Saadah OI, Al-Agha AE, Al Nahdi HM, Bokhary RY, Bin Talib YY, Al-Mughales JA, Al Bokhari SM. Prevalence of celiac disease in children with type 1 diabetes mellitus screened by anti-tissue transglutaminase antibody from Western Saudi Arabia. Saudi Med J. 2012 May;33(5):541-6.

19. Al-Hussaini A, Sulaiman N, Al-Zahrani M, Alenizi A, El Haj I. High prevalence of celiac disease among Saudi children with type 1 diabetes: a prospective cross-sectional study. BMC Gastroenterol. 2012 Dec 23;12:180.

20. Al-Hakami AM. Seroprevalence of coeliac disease in at-risk subjects at the main tertiary hospital, southwest of Saudi Arabia.Arab J Gastroenterol. 2016 Mar;17(1):41-4.

21. Abdulmoein Al-Agha, Ali Ocheltree, Amr Hakeem, Metabolic Control in Children and Adolescents with Insulin-Dependent Diabetes Mellitus at King Abdul-Aziz University Hospital. J Clin Res PediatrEndocrinol 2011;3(4):202-7.

22. Abdulmoein E. Al-Agha, Ali Ocheltree, Amr Hakeem. Occurrence of Microalbuminuria among Children and Adolescents with Insulin-Dependent Diabetes Mellitus. Saudi J Kidney Dis Transpl 2013;24(6):1180-8.

23. Muslim M. Al-Saadi, Sultan A. Meo, Abdul M. Al-Drees, Sarar Mohamed, Shaffi A. Shaikh, Khalid AlRubeaan. Lung functions in poorly controlled type 1 Saudi diabetic children and adolescents. Saudi Med J 2011; Vol. 32 (8): 778-83.

24. Al-Hussaini et al. Prevalence of hepatopathy in type 1 diabetic children BMC Pediatrics 2012, 12:160.

25. Bassam S. Bin-Abbas, Moslah A. Jabari, Sharifah D. Issa, Abdullah H. Al-Fares, Saleh Al-Muhsen. Vitamin D levels in Saudi children with type 1 diabetes. Saudi Med J 2011; Vol. 32 (6): 589-92.

26. Adnan Al Shaikh, Abdullah M. Al Zahrani. Impact of Vitamin D Status on Cardiometabolic Complications among Children and Adolescents with Type 1 Diabetes Mellitus. J Clin Res PediatrEndocrinol 2016;8(1):48-54. 
27. FadiaAlBuhairan. MalihaNasim, Ahlam Al Otaibi, Naila A. Shaheen, Saleh Al Jaser, Ibrahim Al Alwan. Health related quality of life and family impact of type 1 diabetes among adolescents in Saudi Arabia. Diabetes Research and Clinical Practice $114\left(\begin{array}{lllll}2 & 0 & 1 & 6\end{array}\right) 173-9$.

28. Ayman A. Al-Hayek, Asirvatham A. Robert, Huda M. Abbas, Mohamed B. Itani, Abdulghani H. Al-Saeed, Amal E. Juhani, Hanouf S. Al-Goudah, Fahad S. Al-Sabaan. Assessment of health-related quality of life among adolescents with type 1 diabetes mellitus in Saudi Arabia. Saudi Med J 2014; Vol. 35 (7): 712-7.

29. Mohamed A. Abdullah. Epidemiology of Type 1Diabetes Mellitus among Arab children. Saudi Med J 2005; Vol. 26 (6): 911-7.

30. Pinkey JH, Bingley PJ, Sawtell PA, Dunger DB, Gale EA. Presentation and progress of childhood diabetes mellitus: a prospective population-based study. The Bart's-Oxford Study Group. Diabetologia. 1994 Jan;37(1):70-4. 